\title{
Introducing intermediate filaments: from discovery to disease
}

\author{
John E. Eriksson,, ${ }^{1,2}$ Thomas Dechat, ${ }^{3}$ Boris Grin, ${ }^{3}$ Brian Helfand, ${ }^{4}$ Melissa Mendez, ${ }^{3}$ \\ Hanna-Mari Pallari, 1,2,5 and Robert D. Goldman ${ }^{3}$
}

\begin{abstract}
${ }^{1}$ Department of Biology, Åbo Akademi University, Turku, Finland. ${ }^{2}$ Turku Centre for Biotechnology, University of Turku and Åbo Akademi University, Turku, Finland. ${ }^{3}$ Department of Cell and Molecular Biology and ${ }^{4}$ Department of Urology, Northwestern University Medical School, Chicago, Illinois, USA. ${ }^{5}$ Department of Biology, University of Turku, Turku, Finland.
\end{abstract}

\begin{abstract}
It took more than 100 years before it was established that the proteins that form intermediate filaments (IFs) comprise a unified protein family, the members of which are ubiquitous in virtually all differentiated cells and present both in the cytoplasm and in the nucleus. However, during the past 2 decades, knowledge regarding the functions of these structures has been expanding rapidly. Many disease-related roles of IFs have been revealed. In some cases, the molecular mechanisms underlying these diseases reflect disturbances in the functions traditionally assigned to IFs, i.e., maintenance of structural and mechanical integrity of cells and tissues. However, many disease conditions seem to link to the nonmechanical functions of IFs, many of which have been defined only in the past few years.
\end{abstract}

\section{Introduction}

Intermediate filaments (IFs) represent one of the main cytoskeletal systems found in virtually all vertebrate cells. Depending upon the cell type, IFs are composed of different members of the cytoskeletal IF protein family (Figure 1). IF proteins are also present in the nucleus, where they are the main components of the nucleoskeleton.

In this Review we present a historical overview of the discovery and characterization of cytoplasmic and nuclear IFs. Furthermore, we explore many of the features of cytoplasmic IFs that underlie their relevance to disease (Table 1), including their dynamic properties and participation in cytoskeletal crosstalk as well as their roles in signaling, mechanical stabilization, and motility. Although IFs have been studied in various species, such as squid, Xenopus laevis, and Caenorhabditis elegans, we focus here mainly on data obtained from studies in mammalian systems.

\section{Historical overview of IFs}

Early difficulties in the recognition of IFs as a distinct cytoskeletal system. Retrospectively, the first observations of IFs were made by cytologists and histologists during the period when silver staining was used to generate contrast for observations with bright-field microscopy. For example, the structures called tonofibrils that were described in various silver-stained epithelial tissues (1) were likely the same tonofibrils described much later with antibodies directed against keratin (2) and that we now know are composed of densely packed bundles of IFs (see below).

Interest in the composition of natural fibers also stimulated early studies of IFs, which began with X-ray diffraction analyses of human hair, sheep wool, and porcupine quills (3). These studies led to the first descriptions of the $\alpha$-type diffraction patterns generated by keratin IFs. Subsequently, these patterns were shown to represent coiled/coil interactions of 2 or more $\alpha$-helical proteins (4). EM studies provided the first ultrastructural details of bundles of IFs (tonofibrils) in thin sections of wool, hair, and various epithelial

Conflict of interest: The authors have declared that no conflict of interest exists.

Nonstandard abbreviations used: FRAP, fluorescence recovery after photobleaching; GFAP, glial fibrillary acidic protein; IF, intermediate filament; K, keratin; MT, microtubule.

Citation for this article: J. Clin. Invest. 119:1763-1771 (2009). doi:10.1172/JCI38339. cells, including epidermal keratinocytes (5). Over the next 20 years or so, EM revealed fibers with an average diameter of approximately $10 \mathrm{~nm}$ in many cell types - including those comprising skeletal, smooth, and cardiac muscle; those forming epithelial tissues, such as the epidermis; and those found in nerve tissue - as well as in cultured cells such as fibroblasts (6-11). During this period, 10-nm filaments were given many different names, including square filaments, round filaments, neurofilaments, and $\beta$ filaments (12).

In addition to the variety of names, alternate hypotheses regarding the origins of the 10-nm filaments delayed the recognition of IFs as a unique protein family. For example, it was suggested that IFs were an alternately assembled form of microtubules (MTs). This idea arose from EM observations that MT inhibitors, such as colchicine and colcemid, apparently increased the number of $10-\mathrm{nm}$ filaments coincident with MT depolymerization $(13,14)$. However, we now know that after MT depolymerization, some types of IFs retract from the cell surface and reorganize into large bundles around the nucleus $(8,9,15,16)$. At the time, it was suggested that the IFs within the bundles were another polymerized form of tubulin (13, 14). Further confusion regarding the differences between IFs and MTs stemmed from the fact that the major IF protein in fibroblasts, vimentin, has a molecular weight similar to that of tubulin, which made them difficult to separate by gel electrophoresis $(17,18)$. In addition, it was even considered for a brief period that IFs contained a microfilament core $(19,20)$. However, Ishikawa et al. suggested that the 10 -nm filaments they observed in cells from developing muscle might represent a distinct class of cytoskeletal structures, which they called intermediate-sized filaments (7). This suggestion was supported by other studies in nonmuscle cells (8).

By the mid- to late 1970s, several different types of IFs had been isolated, solubilized, and purified by cycles of assembly and disassembly in vitro (21-23). These studies took advantage of the unique properties of IFs, namely, that they are insoluble under physiological conditions and are able to repolymerize after disassembly into their subunits under conditions that would irreversibly denature many proteins. Furthermore, in contrast to actin and tubulin, IFs do not require nucleoside triphosphates such as ATP or GTP for assembly, but rather self assemble under very simple conditions.

Classifying IFs. IFs all share a common tripartite structure, consisting of a highly conserved central $\alpha$-helical rod domain and 
Table 1

Diseases associated with mutations in IF proteins

\section{IF}

Types I and II

Acidic and neutral-basic keratins (129)

\section{Type III}

Desmin (129)

GFAP $(89,129)$

Peripherin (129)

Vimentin (130)

Type IV

Neurofilaments (NF-L, -M, and -H) (129)

\section{Type V}

A-type lamins $(43,61,62)$

\section{B-type lamins $(61,62)$}

Type VI

Bfsp1 (filensin) (129)

Bfsp2 (phakinin or CP49) (129)

\section{Examples of diseases}

Epidermolysis bullosa simplex; epidermolytic hyperkeratosis; Meesman corneal dystrophy; monilethrix; keratodermas; oral white sponge nevus; pachyonychia congenita; pseudofolliculitis barbae; steatocystoma multiplex

Desmin-related myopathy; dilated cardiomyopathy $1 \mathrm{~A}$; dilated cardiomyopathy with conduction system deficits; desmin-related limb-girdle muscular dystrophy; distal myopathy; scapuloperoneal syndrome type Kaeser Alexander disease

Amyotrophic lateral sclerosis 1

Dominant cataract

Various Charcot-Marie-Tooth diseases; Parkinson disease; amyotrophic lateral sclerosis 1; neuronal IF inclusion disease

Hutchinson-Gilford progeria syndrome; atypical Werner syndrome; dilated cardiomyopathy $1 \mathrm{~A}$; familial partial lipodystrophy; limb-girdle muscular dystrophy; CharcotMarie-Tooth disorder type 2B1; mandibuloacral dysplasia; Emery-Dreifuss muscular dystrophy; restrictive dermopathy Acquired partial lipodystrophy; adult-onset leukodystrophy

Autosomal-dominant cataract

Autosomal-recessive cataract

Accumulations of IF proteins have also been described as pathological hallmarks associated with diseases not listed here, such as the keratin-containing Mallory-Denk bodies characteristic of alcoholic cirrhosis and other liver disorders. Bfsp, beaded filament structural protein.

variable $\mathrm{N}$-terminal head and $\mathrm{C}$-terminal tail domains (Figure 1A). As IF research progressed, it became evident that different cell types possessed different types of cytoskeletal IF proteins (24). For instance, epithelial cells contain mainly keratins; muscle cells, desmin; mesenchymal cells, vimentin; and neurons, neurofilaments (Figure 1B). It also became apparent that some cell types express more than one IF protein (Figure 2), and that the expression of IF proteins is frequently developmentally regulated (e.g., vimentin is detected in early developing muscle, but not in terminally differentiated muscle; refs. 25, 26). Because of this, classifying IFs based on cell type-specific expression patterns was difficult. Eventually, similarities in the $\alpha$-helical rod domain sequences and assembly properties of the large number of different keratin proteins prompted the classification of cytoskeletal IFs into 4 major types according to central rod domain amino acid sequences, net acidic charge, and secondary structure predictions (27). According to this classification, type I cytoskeletal IF proteins are the acidic keratin proteins, and type II cytoskeletal IF proteins are the neutral-basic keratin proteins. At least one type I keratin and one type II keratin are needed to form heteropolymeric keratin IFs. Type III IF proteins, which include vimentin and desmin, can form homopolymeric IFs as well as heteropolymeric IFs in combination with other type III or type IV IFs. The type IV IF proteins include nestin, synemin, and the neurofilament triplet proteins.

Type VIFs: the nuclear lamins. The nuclear lamins are the major components of a filamentous layer, the nuclear lamina, that is closely associated with the inner nuclear membrane. This layer was originally described in ultrastructural studies of the protozoans Amoeba proteus $(28,29)$ and Gregarina melanoplus (30), as well as in neurons of the leech Hirudo medicinalis $(31,32)$, and often appeared to have a honeycomb structure. The first descriptions of a fibrous lamina in vertebrate cells came from EM observations of smooth muscle cells obtained from the guinea pig epididymis, intestinal epithelial cells from the Congo eel, and interstitial cells from the cat (33). These studies revealed a 15 - to 20 -nm fibrous lamina between the inner nuclear membrane and the underlying peripheral heterochromatin. Several other laboratories described a similar structure, which became known as the nuclear lamina, in various cell types and species $(34,35)$. Isolation of whole nuclei and of nuclear fractions resistant to detergent and high salt (termed lamina/pore complex fractions) from rat liver revealed the close association of the nuclear lamina with heterochromatin and nuclear pore complexes (36-38). These cell-free preparations also revealed that the nuclear lamina is persistent throughout the entire nuclear periphery as a $15-\mathrm{nm}$ layer $(37,38)$, as had been observed in situ $(33,35)$.

Biochemical analyses revealed that the lamina/pore complex fractions isolated from rat liver contained 3 major polypeptides of approximately 60-70 kDa (37-39). Further biochemical characterization of these proteins, eventually named lamins A, B, and $\mathrm{C}$ according to their descending weights (40), revealed that they were closely related structurally, with lamins A and C sharing extensive sequence homology $(41,42)$. This latter finding suggested that lamin $\mathrm{C}$ might be a cleavage product of lamin $\mathrm{A}$ (41). However, later studies showed that lamins A and C are encoded by different mRNAs, although they are derived from a single gene (LMNA) by alternative splicing (reviewed in ref. 43). Furthermore, it was discovered that lamin A is first expressed as a precursor protein (pre-lamin A), which is posttranslationally processed to become mature lamin A (reviewed in ref. 44). With respect to lamin $B$, it was shown that in vertebrate somatic cells, there are 2 isoforms, lamin B1 and lamin B2, encoded by different genes (43). Similar to lamin A, lamins B1 and B2 are synthesized as precursors and subsequently modified into their mature forms (44). Besides lamins A, C, B1, and B2, other, less abundant, lamin isoforms (lamins A $\Delta 10, \mathrm{C} 2$, and B3) have been described (43). Although at least one lamin isoform appears to be present in all metazoan cells, lamins, at least as we have defined them, seem to be absent from unicellular organisms and from plants (reviewed in refs. 45,46 ). It is also of interest to note that comparison of the gene structures of lamin genes and 
A

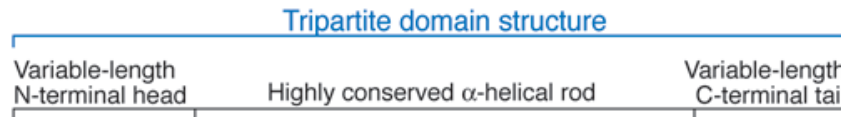

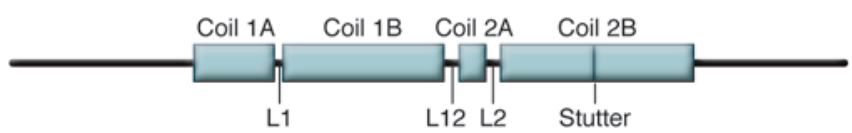

B

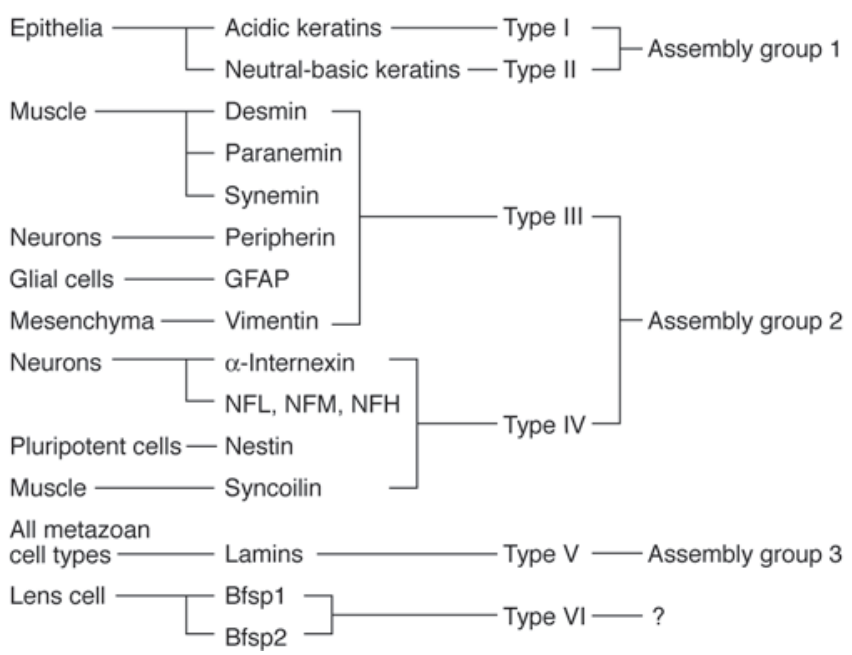

genes encoding cytoplasmic IFs suggests that all IF proteins are derived from a lamin-like progenitor $(47,48)$.

Early cross-linking experiments showed that the nuclear lamins can form oligomeric structures $(42,49)$. Even before the nuclear lamins were recognized as members of the IF protein family, sucrose-gradient density centrifugation and gel-filtration analyses of solubilized fractions of nuclear lamina preparations led to the hypothesis that the basic building blocks of lamin structures were rod-shaped dimers (50). This was supported 3 years later by the finding that lamins belong to the IF protein family, most of which are assembled from rod-shaped, parallel, and in-register dimers (51-55). Because of structural differences between the rod domains of lamins and those of cytoplasmic IF, lamins were designated as type V IF proteins $(27,55)$.

The generation of distinct antibodies specific for each of the lamins led to the observation that lamins A, B, and C localize mainly to

\section{Figure 1}

Overview of the IF protein family. (A) All IF proteins share a tripartite domain structure consisting of a highly conserved $\alpha$-helical central rod domain flanked by variable $\mathrm{N}$-terminal head and $\mathrm{C}$-terminal tail domains. (B) Based on central rod domain amino acid sequences, net acidic charge, and secondary structure predictions, cytoskeletal IF proteins were grouped into 4 sequence homology classes (types I-IV). Nuclear IF proteins, the lamins, constitute a fifth class. The sixth class of IF proteins (also referred to as orphans), beaded filament structural protein 1 (Bfsp1; also known as filensin) and Bfsp2 (also known as phakinin and CP49), form highly specialized IFs found only in the lens of the eye. Based on their abilities to copolymerize, the IF proteins of the 6 types are further subdivided into assembly groups 1-3. NFL, NFM, NFH, low-, middle-, and high-molecular weight neurofilament subunits, respectively.

the periphery of interphase nuclei (Figure 3 ) and that the nuclear lamina is reversibly disassembled during mitosis $(40,56,57)$. Further studies revealed that the disassembly of the nuclear lamina during mitosis requires the phosphorylation of lamins at specific sites by several kinases, including $\mathrm{p} 34^{\mathrm{cdc} 2}$ (reviewed in ref. 58).

Little is known about the actual structure of the nuclear lamina and of lamins in an intact nucleus. Early EM studies of the nuclear lamina of germinal vesicles from Xenopus oocytes revealed an orthogonal network of regularly spaced 10 - to 15 -nm fibers (51), whereas in mammalian cells, only irregular filamentous meshworks have been observed $(59,60)$. Besides their presence at the nuclear periphery, lamins have also been described in the nucleoplasm (Figure 3) (reviewed in ref. 61). Beginning in 1994, a series of studies demonstrated roles for lamins in DNA replication, DNA transcription, DNA repair, cell signaling, cell proliferation, and cell differentiation as well as in the structural, functional, and epigenetic organization of chromatin (61). Furthermore, it has recently been revealed that mutations in LMNA are associated with many different diseases (Table 1) (reviewed in ref. 62). The diversity of lamin-associated processes suggests that the lamins are major components of a nuclear structural framework necessary for the assembly of multiprotein complexes involved in various nuclear functions.

\section{A turning point in IF research: \\ IFs are dynamic cytoarchitectural elements}

With the generation of specific antibodies and the widespread use of immunofluorescence, it soon became obvious that cytoskeletal IF networks formed extensive, complex arrays that pervaded most
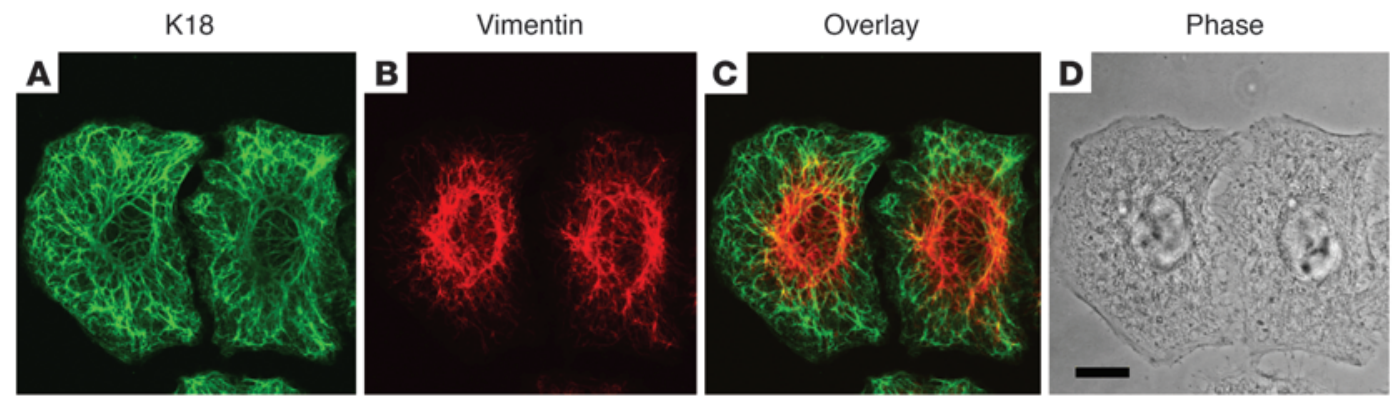

\section{Figure 2}

Coexistence of distinct keratin and vimentin IFs in an individual cell. Human alveolar carcinoma cells were processed for immunofluorescence using antibodies against K18 (A) and vimentin (B). (C) Merged image of A and B. (D) Phase contrast image. Scale bar: $10 \mu \mathrm{M}$. 

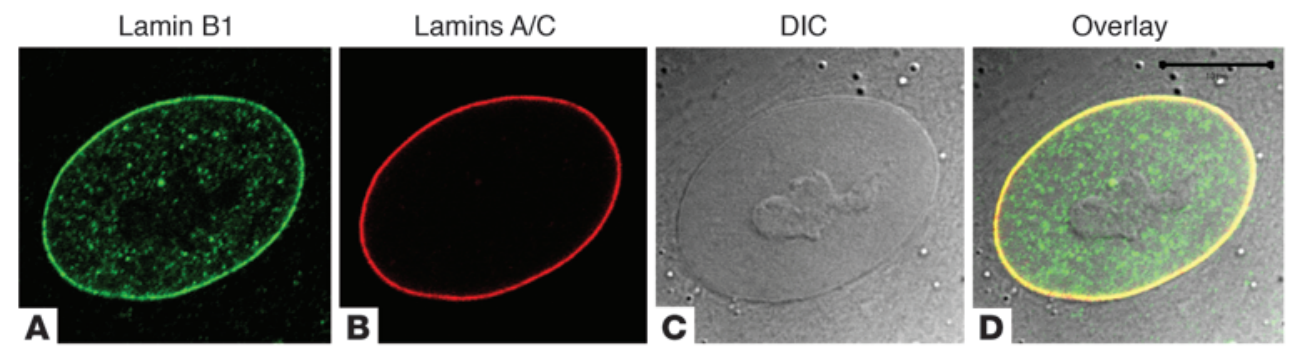

Figure 3

Lamin localization at the nuclear periphery and within the nucleoplasm. Human dermal fibroblasts were processed for indirect immunofluorescence using antibodies against lamins B1 (A) and A/C (B). (C) Differential interference contrast (DIC) image of the cell, shown to visualize the nucleus. (D) Merged image. Scale bar: $10 \mu \mathrm{M}$.

of the cytoplasm of cultured cells $(17,24)$. These IF networks were presumed by numerous investigators to be static, an assumption based on the finding that after extraction of cells in solutions containing relatively high concentrations of salts and detergents, IFs are retained, while most other cellular proteins (including tubulin and actin) are extracted (17). In addition, there are relatively small pools of cytosolic soluble IF proteins (63), which suggested to some that there is very little subunit exchange within IFs.

However, beginning in the late 1980s, studies revealed that ectopically expressed keratin and vimentin readily became incorporated into endogenous IFs through a process termed dynamic subunit exchange $(64,65)$. Similar results were obtained when IF proteins were microinjected into cultured cells $(66,67)$. Soon after, advances in live cell imaging technology permitted assays of IF subunit exchange in vivo using fluorescence recovery after photobleaching (FRAP; refs. 68-70). The results of these FRAP studies confirmed that subunit exchange took place all along the length of vimentin and keratin IFs (Figure 4), providing evidence that they are apolar in vivo. Subsequent studies have demonstrated specific phosphorylation sites that are involved in regulating the dynamic exchange of subunits (reviewed in refs. 71, 72). The phosphate turnover maintaining this dynamic exchange is driven by a high protein phosphatase activity on IFs, something that is also necessary for maintaining the structural integrity of IF polymers $(73,74)$. Further evidence supporting the dynamic properties of IFs have been derived from observations of mesenchymal cells treated with MTdepolymerizing drugs such as colchicine. As MTs disappear, IFs move toward the center of the cell, where they form a perinuclear cap $(8,9,15,16)$. There is also evidence showing that this colchicine-induced IF reorganization requires ATP and microfilaments, which suggests that it is driven by molecular motors (75-77). The redistribution of IFs from a perinuclear cap into the cytoplasmic extensions that form in spreading fibroblasts also suggested early on that IFs are involved in spreading and shape formation (9).

With the use of GFP fusion proteins as reporters for time-lapse observations, it has been shown that individual IFs change shape and propagate wave forms along their lengths $(69,70,78)$. Measuring the rate of subunit turnover on adjacent fibrils in FRAP experiments revealed that filaments move at different speeds relative to each other $(69,70)$. Subunit exchange as well as changes in IF shape require energy, intact MTs, and intact microfilaments $(69,70,78-80)$. Furthermore, the translocation of vimentin IFs is dependent on the MT-based molecular motors kinesin and dynein $(80-82)$. These results confirmed that IFs are highly dynamic struc- tures and brought into focus a new level of functional and regulatory capacity, discussed below (see The switch in the IF paradigm: from structural proteins to multifunctional organizers of cellular processes).

Beginning in the late 1980s and early 1990s, cytoskeletal IF proteins were shown to exist in nonfilamentous forms and in IFs of different lengths. This was especially evident in mitotic cells, in which different types of IFs disassembled into nonfilamentous aggregates that were dispersed throughout the cytoplasm (83-85). Similar nonfilamentous keratin and vimentin particles were seen immediately following their microinjection into cells. At longer times after microinjection, fewer IF particles were seen, and short IFs emerged as particles were incorporated into the endogenous IF network, further demonstrating the dynamic nature of IFs with respect to subunit incorporation $(67,68)$. Direct observations of these different forms of IF were also seen in cells transfected with GFP-tagged IF proteins $(69,70,80,86,87)$. In the early stages of spreading, fibroblasts contain numerous nonfilamentous vimentin particles; these appear to form short IFs, also called squiggles, which then anneal to form long IFs (80). In epithelial cells, keratin also forms particles and squiggles $(70,78,87)$. Many of the vimentin particles and squiggles move rapidly in the cytoplasm, at speeds up to $3 \mu \mathrm{m} / \mathrm{s}$, whereas keratin particles and squiggles move at much slower speeds (approximately $0.3 \mu \mathrm{m} / \mathrm{s}$; refs. 69,87$)$. The movements of the various forms of vimentin and keratin depend on MTs and microfilaments and their associated motors $(70,80,82,87)$, which suggests that IFs may be important mediators of crosstalk among all 3 cytoskeletal systems. Crosstalk among IFs, MTs, and/or microfilaments is also mediated by linker proteins, such as plectin, which can bind to multiple cytoskeletal systems (reviewed in ref. 88). In addition to molecular motors and linker proteins, numerous other proteins have been found to interact with IFs, many of which have welldescribed functions in distinct cellular processes.

\section{The switch in the IF paradigm: from structural proteins to multifunctional organizers of cellular processes}

Although it was clear from the above-mentioned studies that, similar to the other cytoskeletal elements, IFs are highly dynamic structures, the prevailing view of IFs during the 1990s was still that their role was primarily to maintain the structural and mechanical integrity of cells. However, this view began to change, especially with the realization that many of the diseases associated with mutations in the genes encoding IF proteins and aberrations in IF proteins could not easily be related to structural functions, 

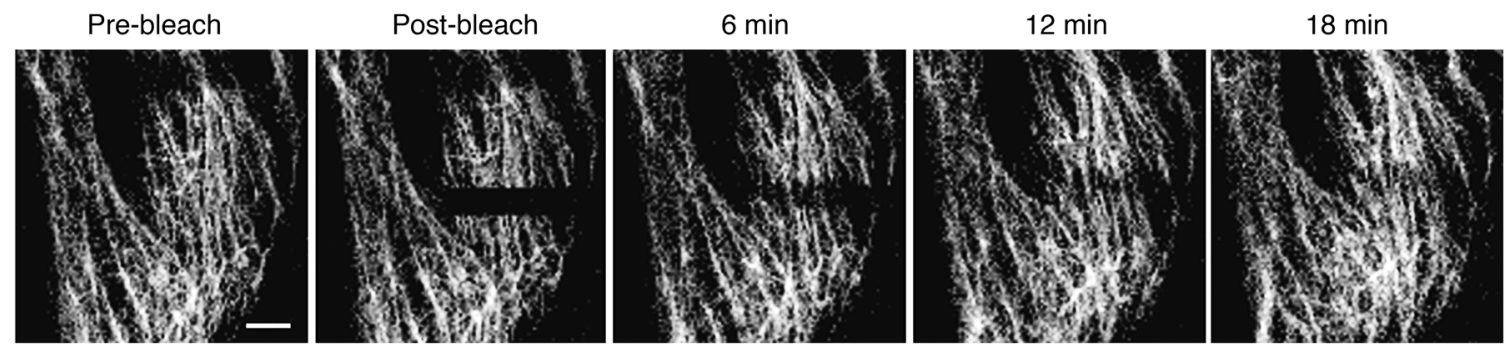

Figure 4

Vimentin subunit exchange as determined by FRAP. After photobleaching (post-bleach, shown by the dark area), the exchange of GFP-tagged vimentin protein subunits within IFs occurs over the length of the bleached zone. Scale bar: $10 \mu \mathrm{M}$.

but rather pointed toward involvement in a broader spectrum of functions, such as organizing cytoplasmic architecture, signaling, and/or regulating transcription (Table 1). This more recent perception is strongly supported by results obtained from studies of laminopathies, a group of diseases resulting from mutations in the genes encoding nuclear lamins. Individuals with these mutations exhibit a broad range of symptoms, from metabolic disturbances to rapidly accelerated aging in children (62). A dramatic example is Alexander disease, which is a rare, neurodegenerative disorder harming the growth of the myelin sheath (89). It is caused by mutations in glial fibrillary acidic protein (GFAP) and characterized by the formation of cytoplasmic GFAP inclusions, termed Rosenthal fibers, in astrocytes. Alterations of the myelin sheath have been suggested to result from the astrocyte dysfunction and disturbed interactions between astrocytes and other specialized brain cells (89). Furthermore, the role of IFs in maintaining cellular homeostasis has been well exemplified by numerous studies revealing that mutations in keratin 8 (K8) or K18 cause or predispose to various liver diseases (90). Cytoprotective, nonmechanical functions of keratins include protection from apoptosis.

Vimentin is the major IF protein found in mesenchymal cells. It is familiar to many researchers, as it is frequently used as a developmental marker of cells and tissues. The advances in cell biology research on vimentin are an example of how the knowledge of novel IF functions has evolved. Vimentin shows a very high degree of sequence homology among species $(91,92)$, implying important and evolutionary conserved physiological roles. However, until recently, the functions of this IF protein remained elusive, as the phenotype of vimentin-deficient mice seemed to be relatively mild (93). More recently, detailed analyses of these mice have revealed significant defects pointing to involvement of vimentin in several cellular functions, including regulation of the morphology of glial cells (94). In addition, vimentin-knockout fibroblasts have deficiencies in motility and directional migration that affect wound healing and fibroblast migration $(95,96)$. Vimentin also has a role in the mechanotransduction of shear stress, as reflected by effects on the resistance of arteries (97). Furthermore, the homing, transmigration, and extravasation of lymphocytes and the integrity of the vascular endothelium are severely affected by a lack of vimentin (98). Subsequent studies shed light on the molecular mechanisms underlying these vimentin-related defects, as vimentin has been shown to participate in a number of critical cellular processes related to the organization and regulation of proteins involved in adhesion, migration, and cell signaling. These functions explain well the observed defects in vimentin-deficient mice. These and other novel functions of IFs are further highlighted here: the examples below are not intended to be comprehensive, but rather present illustrative models of how IFs may function in regulating cellular functions and signal processing (for details on the signaling-related functions of IFs, see refs. 71, 99-102).

IFs as signaling organizers. There is now ample evidence that IFs can act as a scaffold for and as functional determinants of signaling molecules, such as kinases $(71,99-102)$. In many cases, posttranslational modifications by phosphorylation or glycosylation act as regulators of the IF-related signaling functions, as they regulate IF interactions with individual signaling proteins and affect the dynamic properties of the IFs, which in turn has an effect on these interactions $(71,100,102)$.

An example of an IF-kinase interaction is the interaction of vimentin with the RAF-1/RhoA signaling pathway $(103,104)$, which, through Rho-binding kinases (ROKs), is involved in mediating actin dynamics and focal adhesion formation (105). Activated ROK $\alpha$ phosphorylates vimentin, which leads to filament collapse and consequent release of ROK $\alpha$ from the vimentin IFs and its translocation to the periphery of the cell (106). Thus, the presence and organization of vimentin affects the outcome of RhoA-mediated signaling.

The cytoprotective effects of IFs are related to their mechanical properties. However, cytoprotection may also stem from the capacity of IFs to interact with signaling pathways involved in determining cell survival. For example, the IF protein nestin forms a scaffold with cyclin-dependent kinase 5 (Cdk5; ref. 107). In this way, nestin regulates the apoptosis-inducing activity of Cdk5 during oxidant-induced cell death (108). This function could be related to a cytoprotective role for nestin in asymmetric cell division of neuronal stem cells, during which one daughter cell survives and the other undergoes apoptosis. The surviving daughter cell has high nestin content, in contrast to the apoptotic cell, which is low in nestin content but has large amounts of the proapoptotic protein Par-4 (109). Similarly, K8 sequesters the proapoptotic protein JNK after death receptor stimulation (110). This interaction seems to prevent JNK from phosphorylating proapoptotic transcription factor targets, as c-Jun phosphorylation was clearly shown to be reduced under these conditions (110).

IFs may also regulate survival signaling by transport of stressinduced signaling molecules. This functional modality does not require polymer structures, but relies upon nonfilamentous IF precursors. It has been shown that vimentin IF precursors are able to travel along MTs with the help of kinesin and dynein motor proteins, transporting cellular cargo by "walking" on MT 
tracks (reviewed in ref. 111); moreover, these precursors carry along signaling molecules critical for survival processes. This transport mechanism is activated upon nerve injury, when soluble vimentin subunits transport phosphorylated ERK1 and ERK2 from the site of the axonal lesion to the nerve cell body (112). Phosphorylated ERK1/2 is linked by means of vimentin to the nuclear import factor importin, which complexes with dynein and is thereby subjected to dynein-mediated retrograde transport toward the nucleus. This is thought to be a prime reason for the elevated vimentin levels observed specifically in injured neurons (112), as vimentin is then used as a long-distance messenger for the stress-mediated signaling.

In addition to the regulation of the cytoplasmic signaling components associated with cell death and/or survival, IFs can influence cell fate by directly controlling death receptor complexes. In particular, K8/K18 filaments have been described to protect liver hepatocytes during apoptosis-promoting stress conditions, either by regulating the targeting of death receptors at the cell surface (120) or by controlling the formation of the death-inducing signaling complex (DISC) through protein sequestration and thereby controlling downstream signaling (113-115). A similar regulatory role based on the sequestration of DISC components has been suggested for hair follicle K17 in the modulation of TNF- $\alpha$ signaling (116). The importance of IFs has been also observed downstream in death receptor pathways, since keratins have been suggested to form platforms for caspase activation, to limit the intracellular distribution of active caspases (117), and to regulate the ability of death receptor-associated proteins to activate survival-related signaling pathways (118).

Members of the 14-3-3 protein family typically modulate the functions of signaling proteins through direct phosphorylationdependent interactions. IFs have been suggested to have a number of roles in regulating 14-3-3 functions. For example, selective phosphorylation of K18 Ser33 during mitosis promotes the interaction between 14-3-3 $\zeta$ and K18, thereby changing K18 organization and distribution $(119,120)$. Absence of K8, and thereby loss of K8/K18 IFs, shifts 14-3-3 $\zeta$ distribution toward accumulation in the nucleus, arresting cells in $\mathrm{S} / \mathrm{G}_{2}$ phase. The K18/14-3-3 interaction seems to regulate $14-3-3$ binding to phosphorylated Cdc25, a crucial mitotic checkpoint regulator. $\mathrm{Cdc} 25$ - the main function of which is to remove the inhibitory phosphate residues from target cyclin-dependent kinases and thereby regulate progression of the cell cycle - can be suppressed by interaction with 14-3-3, which prohibits $\mathrm{Cdc} 25$ from interacting with its specific cyclindependent kinase. Whether 14-3-3 is bound to K18 affects the ability of 14-3-3 to interact with Cdc25. This implies that the keratin network has the capacity to prevent uncontrolled and potentially harmful 14-3-3 interactions (121).

Another intriguing example of IF/14-3-3 interactions is the interaction between K17 and 14-3-30. Cell growth requires strict orchestration during development and in response to various injuries. K17, an IF protein rapidly induced in wounded stratified epithelia, has been shown to regulate cell growth through binding to 14-3-3o. The interaction with $\mathrm{K} 17$ regulates the Akt/mammalian target of rapamycin (Akt/mTOR) signaling pathway, which is key in the control of protein synthesis and thereby the growth of cells, tissues, and organisms (122). These findings revealed an interesting feedback loop between a wound-induced IF and a signaling pathway that regulates cell growth and size by controlling protein synthesis during the healing process.
IFs as buffers of cellular stress. Beyond their roles in maintaining cell integrity and serving as regulators for stress-activated kinases, IFs have been assigned roles as stress proteins. For example, K8 and $\mathrm{K} 18$ are phosphorylated during different forms of cell stress (reviewed in ref. 100). It has been shown that the K18 Ser52 $\rightarrow$ Ala52 (S52A) mutation, which makes this residue phosphorylation deficient, predisposes transgenic mice to hepatotoxic injury, leading to the postulation that this keratin phosphorylation has a hepatoprotective effect (123). The idea of protective keratin phosphorylation was recently shown to be applicable in the context of the human liver disease-associated mutation K8 Gly61 $\rightarrow$ Cys61 (G61C; ref. 124). Transgenic mice overexpressing K8 with this mutation were more sensitive to liver injury and apoptosis than were wild-type mice (124). Interestingly, the G61C mutation inhibited K8 phosphorylation at Ser73, which is targeted by stress-activated protein kinases, such as p38 kinase and JNK (124). Although the susceptibility to injury of mice overexpressing a Ser73 $\rightarrow$ Ala73 (S73A) mutant was the same as that of mice overexpressing the G61C mutant, it was concluded that keratins protect tissues from injuries by serving as a phosphate "sponge" that absorbs stressinduced kinase activity (124). The amino acid sequence surrounding Ser73 in K8 resembles phosphorylation motifs found in other type II keratins that become phosphorylated during stress (125), which indicates that this type of phosphate buffering could be a general phenomenon of type II keratins (124).

IFs and migration. Focal contacts participate in the movement of cells on a substratum. While focal contacts are considered to anchor actin filaments, vimentin has also been reported to accumulate at adhesion sites at focal adhesions (126). These structures are termed vimentin-associated matrix adhesions (VMAs). Because VMAs assemble in actively migrating cells, it has been suggested that they support motility (126). The architecture of focal contacts is disturbed in vimentin-deficient fibroblasts (96), which supports a role for vimentin in regulating focal adhesions. Further support of this role came from the demonstration that the vimentin cytoskeleton regulates focal contact size and helps to stabilize cell-ECM adhesions in endothelial cells (127). Interestingly, the transcellular migration of lymphocytes through vimentin-deficient endothelial cell barriers is severely impaired when endothelial cells or lymphocytes lack vimentin. It was shown that this effect was due to the disturbed distribution of adhesion molecules (integrin $\beta_{1}$ in lymphocytes and both ICAM1 and VCAM1 in endothelial cells) in the vimentin-deficient cells (98). Interestingly, vimentin is thought to be an important organizer of integrins, as it participates in the PKCE-mediated trafficking of integrins to the plasma membrane. In this way, vimentin modulates cell motility that is dependent on the cycling of adhesion site proteins (128).

\section{Conclusions}

The literature reveals very early published descriptions of IFs, but it took a long time for the realization that this is a very large family of proteins with highly conserved common structural and biochemical features that determine their assembly into polymers. After the paradigm of IFs as a unified protein family was established, it still took many years before it was realized that these proteins form highly dynamic structures in the same way that other cytoskeletal elements do. Recent developments have shed light on numerous disease-related functions of IFs, many of which are likely to be associated with their nonmechanical functions (Table 1). These 
broad-ranging, previously unknown functions of cytoplasmic IF proteins all relate to the capacity of IFs to actively sequester, position, or act as scaffolds for signaling molecules, including developmentally active and cell fate-determining kinases. The interaction with stress-activated kinases also makes them highly suitable to act as phosphate sponges in stressed cells. IFs are in rich supply in most cells, making them efficient both as stress buffers and as signaling scaffolds, even for high-abundance signaling molecules such as 14-3-3. While the knowledge of the functions of these proteins has rapidly accumulated during the past 2 decades, it seems more than likely that many important functions of IFs are yet to be discovered. In light of the numerous recently discovered signaling- related functions of IFs, it seems probable that many of these functions will be related to a broad range of signaling pathways that are important for normal homeostasis as well as for developmental and stress-related processes. Given the importance of signaling in disease onset and progress, deciphering these functions will either reveal direct IF-related links to disease processes or pave the way toward a better understanding of different disease processes.

Address correspondence to: John Eriksson, Department of Biology, Åbo Akademi University, Tykistökatu 6B, FI-20520 Turku, Finland. Phone: 358-2-215-3313; Fax: 333-2-215-4748; E-mail: john.eriksson@abo.fi.
1. Wilson, E.B. 1928. The cell in development and heredity. 3rd edition. Macmillan Company. New York, New York, USA. 1232 pp.

2. Jones, J.C., and Goldman, R.D. 1985. Intermediate filaments and the initiation of desmosome assembly. J. Cell Biol. 101:506-517.

3. Astbury, W.T., and Street, A. 1930. X-ray studies of the structure of hair, wool, and related fibres. I. General. Philos. Trans. R. Soc. Lond. A. 230:75-101.

4. Crick, F.H. 1952. Is alpha-keratin a coiled coil? Nature. 170:882-883.

5. Fraser, R.D., and Macrae, T.P. 1961. The molecular configuration of alpha-keratin. J. Mol. Biol. 3:640-647.

6. Schmitt, F.O. 1968. Fibrous proteins - neuronal organelles. Proc. Natl. Acad. Sci. U. S. A. 60:1092-1101.

7. Ishikawa, H., Bischoff, R., and Holtzer, H. 1968. Mitosis and intermediate-sized filaments in developing skeletal muscle. J. Cell Biol. 38:538-555.

8. Goldman, R.D. 1971. The role of three cytoplasmic fibers in BHK-21 cell motility. I. Microtubules and the effects of colchicine. J. Cell Biol. 51:752-762.

9. Goldman, R.D., and Follett, E.A. 1970. Birefringent filamentous organelle in BHK-21 cells and its possible role in cell spreading and motility. Science. 169:286-288.

10. Rice, R.V., Moses, J.A., McManus, G.M., Brady, A.C., and Blasik, L.M. 1970. The organization of contractile filaments in a mammalian smooth muscle. J. Cell Biol. 47:183-196.

11. Cooke, P. 1976. A filamentous cytoskeleton in vertebrate smooth muscle fibers. J. Cell Biol. 68:539-556.

12. Eriksson, A., and Thornell, L.E. 1979. Intermediate (skeletin) filaments in heart Purkinje fibers. A correlative morphological and biochemical identification with evidence of a cytoskeletal function. J. Cell Biol. 80:231-247.

13. Wisniewski, H., Shelanski, M.L., and Terry, R.D. 1968. Effects of mitotic spindle inhibitors on neurotubules and neurofilaments in anterior horn cells. J. Cell Biol. 38:224-229.

14. Holmes, K.V., and Choppin, P.W. 1968. On the role of microtubules in movement and alignment of nuclei in virus-induced syncytia. J. Cell Biol. 39:526-543.

15. Blose, S.H., and Chacko, S. 1976. Rings of intermediate $(100 \mathrm{~A})$ filament bundles in the perinuclear region of vascular endothelial cells. Their mobilization by colcemid and mitosis. J. Cell Biol. 70:459-466.

16. Blose, S.H., Meltzer, D.I., and Feramisco, J.R. 1984. 10-nm filaments are induced to collapse in living cells microinjected with monoclonal and polyclonal antibodies against tubulin. J. Cell Biol. 98:847-858.

17. Starger, J.M., Brown, W.E., Goldman, A.E., and Goldman, R.D. 1978. Biochemical and immunological analysis of rapidly purified $10-\mathrm{nm}$ filaments from baby hamster kidney (BHK-21) cells. J. Cell Biol. 78:93-109.

18. Starger, J.M., and Goldman, R.D. 1977. Isolation and preliminary characterization of $10-\mathrm{nm}$ fila- ments from baby hamster kidney (BHK-21) cells. Proc. Natl. Acad. Sci. U. S. A. 74:2422-2426.

19. Buckley, I.K., Raju, T.R., and Stewart, M. 1978. Heavy meromyosin labeling of intermediate filaments in cultured connective tissue cells. J. Cell Biol. 78:644-652.

20. Buckley, I.K., Raju, T.R., and Stewart, M. 1981. Claims that intermediate filaments contain F-actin are unwarranted. J. Cell Biol. 90:309-311.

21. Zackroff, R.V., and Goldman, R.D. 1980. In vitro reassembly of squid brain intermediate filaments (neurofilaments): purification by assembly-disassembly. Science. 208:1152-1155.

22. Zackroff, R.V., and Goldman, R.D. 1979. In vitro assembly of intermediate filaments from baby hamster kidney (BHK-21) cells. Proc. Natl. Acad. Sci. U. S. A. 76:6226-6230.

23. Steinert, P.M., and Gullino, M.I. 1976. Bovine epidermal keratin filament assembly in vitro. Biochem. Biophys. Res. Commun. 70:221-227.

24. Franke, W.W., Schmid, E., Osborn, M., and Weber, K. 1978. Different intermediate-sized filaments distinguished by immunofluorescence microscopy. Proc. Natl. Acad. Sci. U. S. A. 75:5034-5038.

25. Gard, D.L., Bell, P.B., and Lazarides, E. 1979. Coexistence of desmin and the fibroblastic intermediate filament subunit in muscle and nonmuscle cells: identification and comparative peptide analysis. Proc. Natl. Acad. Sci. U. S. A. 76:3894-3898.

26. Granger, B.L., and Lazarides, E. 1979. Desmin and vimentin coexist at the periphery of the myofibril Z disc. Cell. 18:1053-1063.

27. Steinert, P.M., and Roop, D.R. 1988. Molecular and cellular biology of intermediate filaments. Annu. Rev. Biochem. 57:593-625.

28. Mercer, E.H. 1959. An electron microscopic study of Amoeba proteus. Proc. R. Soc. Lond. B Biol. Sci. 150:216-232.

29. Pappas, G.D. 1956. The fine structure of the nuclear envelope of Amoeba proteus. J. Biophys. Biochem. Cytol. 2:431-434.

30. Beams, H.W., Tahmisian, T.N., Devine, R., and Anderson, E. 1957. Ultrastructure of the nuclear membrane of a gregarine parasitic in grasshoppers. Exp. Cell Res. 13:200-204.

31. Coggeshall, R.E., and Fawcett, D.W. 1964. The fine structure of the central nervous system of the leech, Hirudo medicinalis. J. Neurophysiol. 27:229-289.

32. Gray, E.G., and Guillery, R.W. 1963. An electron microscopical study of the ventral nerve cord of the leech. Z. Zellforsch. Mikrosk. Anat. 60:826-849.

33. Fawcett, D.W. 1966. On the occurrence of a fibrous lamina on the inner aspect of the nuclear envelope in certain cells of vertebrates. Am J. Anat. 119:129-145.

34. Patrizi, G., and Poger, M. 1967. The ultrastructure of the nuclear periphery. The zonula nucleum limitans. J. Ultrastruct. Res. 17:127-136.

35. Oryschak, A.F., Ghadially, F.N., and Bhatnagar, R. 1974. Nuclear fibrous lamina in the chondrocytes of articular cartilage. J. Anat. 118:511-515.

36. Barton, A.D., Kisieleski, W.E., Wassermann, F., and Mackevicius, F. 1971. Experimental modification of structures at the periphery of the liver cell nucleus. Z. Zellforsch. Mikrosk. Anat. 115:299-306.

37. Aaronson, R.P., and Blobel, G. 1975. Isolation of nuclear pore complexes in association with a lamina. Proc. Natl. Acad. Sci. U. S. A. 72:1007-1011.

38. Dwyer, N., and Blobel, G. 1976. A modified procedure for the isolation of a pore complex-lamina fraction from rat liver nuclei. J. Cell Biol. 70:581-591.

39. Berezney, R., and Coffey, D.S. 1974. Identification of a nuclear protein matrix. Biochem. Biophys. Res. Commun. 60:1410-1417.

40. Gerace, L., and Blobel, G. 1980. The nuclear envelope lamina is reversibly depolymerized during mitosis. Cell. 19:277-287.

41. Cochran, D.L., Yeoman, L.C., Egle, P.M., and Shelton, K.R. 1979. Comparison of the major polypeptides of the erythrocyte nuclear envelope. J. Supramol. Struct. 10:405-418.

42. Kaufmann, S.H., Gibson, W., and Shaper, J.H. 1983. Characterization of the major polypeptides of the rat liver nuclear envelope. J. Biol. Chem. 258:2710-2719.

43. Broers, J.L., Ramaekers, F.C., Bonne, G., Yaou, R.B., and Hutchison, C.J. 2006. Nuclear lamins: laminopathies and their role in premature ageing. Physiol. Rev. 86:967-1008.

44. Rusinol, A.E., and Sinensky, M.S. 2006. Farnesylated lamins, progeroid syndromes and farnesyl transferase inhibitors. J. Cell Sci. 119:3265-3272.

45. Meier, I. 2007. Composition of the plant nuclear envelope: theme and variations. J. Exp. Bot. 58:27-34.

46. Melcer, S., Gruenbaum, Y., and Krohne, G. 2007. Invertebrate lamins. Exp. Cell Res. 313:2157-2166.

47. Dodemont, H., Riemer, D., and Weber, K. 1990. Structure of an invertebrate gene encoding cytoplasmic intermediate filament (IF) proteins: implications for the origin and the diversification of IF proteins. EMBO J. 9:4083-4094.

48. Doring, V., and Stick, R. 1990. Gene structure of nuclear lamin LIII of Xenopus laevis; a model for the evolution of IF proteins from a lamin-like ancestor. EMBO J. 9:4073-4081.

49. Shelton, K.R., Guthrie, V.H., and Cochran, D.L. 1982. Oligomeric structure of the major nuclear envelope protein lamin B. J. Biol. Chem. 257:4328-4332.

50. Havre, P.A., and Evans, D.R. 1983. Disassembly and characterization of the nuclear pore complex-lamina fraction from bovine liver nuclei. Biochemistry. 22:2852-2860.

51. Aebi, U., Cohn, J., Buhle, L., and Gerace, L. 1986. The nuclear lamina is a meshwork of intermediatetype filaments. Nature. 323:560-564.

52. Fisher, D.Z., Chaudhary, N., and Blobel, G. 1986. cDNA sequencing of nuclear lamins $A$ and $C$ reveals primary and secondary structural homology to intermediate filament proteins. Proc. Natl. Acad. Sci. U. S. A. 83:6450-6454.

53. Goldman, A.E., Maul, G., Steinert, P.M., Yang, H.Y., and Goldman, R.D. 1986. Keratin-like proteins that coisolate with intermediate filaments of BHK-21 cells are nuclear lamins. Proc. Natl. Acad. Sci. U. S. A. 83:3839-3843.

54. McKeon, F.D., Kirschner, M.W., and Caput, D. 
1986. Homologies in both primary and secondary structure between nuclear envelope and intermediate filament proteins. Nature. 319:463-468.

55. Parry, D.A., Conway, J.F., and Steinert, P.M. 1986. Structural studies on lamin. Similarities and differences between lamin and intermediate-filament proteins. Biochem. J. 238:305-308.

56. Gerace, L., Blum, A., and Blobel, G. 1978. Immunocytochemical localization of the major polypeptides of the nuclear pore complex-lamina fraction. Interphase and mitotic distribution. J. Cell Biol. 79:546-566.

57. Krohne, G., Franke, W.W., Ely, S., D’Arcy, A., and Jost, E. 1978. Localization of a nuclear envelopeassociated protein by indirect immunofluorescence microscopy using antibodies against a major polypeptide from rat liver fractions enriched in nuclear envelope-associated material. Cytobiologie. 18:22-38.

58. Fields, A.P., and Thompson, L.J. 1995. The regulation of mitotic nuclear envelope breakdown: a role for multiple lamin kinases. Prog. Cell Cycle Res. 1:271-286.

59. Shimi, T., et al. 2008. The A- and B-type nuclear lamin networks: microdomains involved in chromatin organization and transcription. Genes Dev 22:3409-3421.

60. Schermelleh, L., et al. 2008. Subdiffraction multicolor imaging of the nuclear periphery with 3D structured illumination microscopy. Science. 320:1332-1336.

61. Dechat, T., et al. 2008. Nuclear lamins: major factors in the structural organization and function of the nucleus and chromatin. Genes Dev. 22:832-853.

62. Worman, H.J., Fong, L.G., Muchir, A., and Young, S.G. 2009. Laminopathies and the long strange trip from basic cell biology to therapy. J. Clin. Invest. 119:1825-1836.

63. Soellner, P., Quinlan, R.A., and Franke, W.W. 1985 Identification of a distinct soluble subunit of an intermediate filament protein: tetrameric vimentin from living cells. Proc. Natl. Acad. Sci. U. S. A 82:7929-7933.

64. Albers, K., and Fuchs, E. 1989. Expression of mutant keratin cDNAs in epithelial cells reveals possible mechanisms for initiation and assembly of intermediate filaments. J. Cell Biol. 108:1477-1493.

65. Ngai, J., Coleman, T.R., and Lazarides, E. 1990 Localization of newly synthesized vimentin subunits reveals a novel mechanism of intermediate filament assembly. Cell. 60:415-427.

66. Vikstrom, K.L., Borisy, G.G., and Goldman, R.D. 1989. Dynamic aspects of intermediate filament networks in BHK-21 cells. Proc. Natl. Acad. Sci.U. S. A. 86:549-553.

67. Miller, R.K., Vikstrom, K., and Goldman, R.D. 1991. Keratin incorporation into intermediate filament networks is a rapid process. J. Cell Biol. 113:843-855.

68. Vikstrom, K.L., Lim, S.S., Goldman, R.D., and Borisy, G.G. 1992. Steady state dynamics of intermediate filament networks. J. Cell Biol. 118:121-129.

69. Yoon, K.H., et al. 2001. Insights into the dynamic properties of keratin intermediate filaments in living epithelial cells. J. Cell Biol. 153:503-516.

70. Yoon, M., Moir, R.D., Prahlad, V., and Goldman, R.D. 1998. Motile properties of vimentin intermediate filament networks in living cells. J. Cell Biol. 143:147-157

71. Hyder, C.L., Pallari, H.M., Kochin, V., and Eriksson, J.E. 2008. Providing cellular signposts - post-translational modifications of intermediate filaments. FEBS Lett. 582:2140-2148.

72. Sihag, R.K., Inagaki, M., Yamaguchi, T., Shea, T.B., and Pant, H.C. 2007. Role of phosphorylation on the structural dynamics and function of types III and IV intermediate filaments. Exp. Cell Res. 313:2098-2109.

73. Eriksson, J.E., Opal, P., and Goldman, R.D. 1992.
Intermediate filament dynamics. Curr. Opin. Cell Biol. 4:99-104.

74. Toivola, D.M., Goldman, R.D., Garrod, D.R., and Eriksson, J.E. 1997. Protein phosphatases maintain the organization and structural interactions of hepatic keratin intermediate filaments. J. Cell Sci. 110:23-33.

75. Rodionov, V.I., et al. 1993. Microtubule-dependent control of cell shape and pseudopodial activity is inhibited by the antibody to kinesin motor domain. J. Cell Biol. 123:1811-1820.

76. Hollenbeck, P.J., Bershadsky, A.D., Pletjushkina, O.Y., Tint, I.S., and Vasiliev, J.M. 1989. Intermediate filament collapse is an ATP-dependent and actindependent process. J. Cell Sci. 92:621-631.

77. Tint, I.S., Hollenbeck, P.J., Verkhovsky, A.B., Surgucheva, I.G., and Bershadsky, A.D. 1991. Evidence that intermediate filament reorganization is induced by ATP-dependent contraction of the actomyosin cortex in permeabilized fibroblasts. J. Cell Sci. 98:375-384.

78. Windoffer, R., and Leube, R.E. 1999. Detection of cytokeratin dynamics by time-lapse fluorescence microscopy in living cells. J. Cell Sci. 112:4521-4534.

79. Ho, C.L., Martys, J.L., Mikhailov, A., Gundersen, G.G., and Liem, R.K. 1998. Novel features of intermediate filament dynamics revealed by green fluorescent protein chimeras. J. Cell Sci. 111:1767-1778.

80. Prahlad, V., Yoon, M., Moir, R.D., Vale, R.D., and Goldman, R.D. 1998. Rapid movements of vimentin on microtubule tracks: kinesin-dependent assembly of intermediate filament networks. J. Cell Biol. 143:159-170.

81. Gyoeva, F.K., and Gelfand, V.I. 1991. Coalignment of vimentin intermediate filaments with microtubules depends on kinesin. Nature. 353:445-448.

82. Helfand, B.T., Mikami, A., Vallee, R.B., and Goldman, R.D. 2002. A requirement for cytoplasmic dynein and dynactin in intermediate filament network assembly and organization. J. Cell Biol. 157:795-806

83. Chou, Y.H., Rosevear, E., and Goldman, R.D. 1989 Phosphorylation and disassembly of intermediate filaments in mitotic cells. Proc. Natl. Acad. Sci.U. S. A. 86:1885-1889

84. Jones, J.C., Goldman, A.E., Yang, H.Y., and Goldman, R.D. 1985. The organizational fate of intermediate filament networks in two epithelial cell types during mitosis. J. Cell Biol. 100:93-102.

85. Lane, E.B., Goodman, S.L., and Trejdosiewicz, L.K 1982. Disruption of the keratin filament network during epithelial cell division. EMBOJ. 1:1365-1372.

86. Windoffer, R., Woll, S., Strnad, P., and Leube, R.E. 2004. Identification of novel principles of keratin filament network turnover in living cells. Mol. Biol. Cell. 15:2436-2448.

87. Woll, S., Windoffer, R., and Leube, R.E. 2005. Dissection of keratin dynamics: different contributions of the actin and microtubule systems. Eur. J. Cell Biol. 84:311-328.

88. Sonnenberg, A., Rojas, A.M., and de Pereda, J.M. 2007. The structure of a tandem pair of spectrin repeats of plectin reveals a modular organization of the plakin domain. J. Mol. Biol. 368:1379-1391.

89. Quinlan, R.A., Brenner, M., Goldman, J.E., and Messing, A. 2007. GFAP and its role in Alexander disease. Exp. Cell Res. 313:2077-2087.

90. Ku, N.O., Strnad, P., Zhong, B.H., Tao, G.Z., and Omary, M.B. 2007. Keratins let liver live: Mutations predispose to liver disease and crosslinking generates Mallory-Denk bodies. Hepatology. 46:1639-1649.

91. Herrmann, H., Fouquet, B., and Franke, W.W. 1989. Expression of intermediate filament proteins during development of Xenopus laevis. I. cDNA clones encoding different forms of vimentin. Development. 105:279-298.

92. Schaffeld, M., Herrmann, H., Schultess, J., and Markl, J. 2001. Vimentin and desmin of a carti- laginous fish, the shark Scyliorhinus stellaris: sequence, expression patterns and in vitro assembly. Eur. J. Cell Biol. 80:692-702.

93. Colucci-Guyon, E., et al. 1994. Mice lacking vimentin develop and reproduce without an obvious phenotype. Cell. 79:679-694.

94. Colucci-Guyon, E., Gimenez, Y.R.M., Maurice, T. Babinet, C., and Privat, A. 1999. Cerebellar defect and impaired motor coordination in mice lacking vimentin. Glia. 25:33-43.

95. Eckes, B., et al. 2000. Impaired wound healing in embryonic and adult mice lacking vimentin. J. Cell Sci. 113:2455-2462

96. Eckes, B., et al. 1998. Impaired mechanical stability, migration and contractile capacity in vimentindeficient fibroblasts. J. Cell Sci. 111:1897-1907.

97. Henrion, D., et al. 1997. Impaired flow-induced dilation in mesenteric resistance arteries from mice lacking vimentin. J. Clin. Invest. 100:2909-2914.

98. Nieminen, M., et al. 2006. Vimentin function in lymphocyte adhesion and transcellular migration. Nat. Cell Biol. 8:156-162.

99. Ivaska, J., Pallari, H.M., Nevo, J., and Eriksson, J.E. 2007. Novel functions of vimentin in cell adhesion, migration, and signaling. Exp. Cell Res. 313:2050-2062.

100.Omary, M.B., Ku, N.O., Tao, G.Z., Toivola, D.M., and Liao, J. 2006. "Heads and tails" of intermediate filament phosphorylation: multiple sites and functional insights. Trends Biochem. Sci. 31:383-394.

101. Pallari, H.M., and Eriksson, J.E. 2006. Intermediate filaments as signaling platforms. Sci. STKE. 2006:pe53.

102. Toivola, D.M., Tao, G.Z., Habtezion, A., Liao, J., and Omary, M.B. 2005. Cellular integrity plus: organelle-related and protein-targeting functions of intermediate filaments. Trends Cell Biol. 15:608-617.

103. Ehrenreiter, K., et al. 2005. Raf-1 regulates Rho signaling and cell migration. J. Cell Biol. 168:955-964.

104.Janosch, P., et al. 2000. The Raf-1 kinase associates with vimentin kinases and regulates the structure of vimentin filaments. FASEBJ. 14:2008-2021.

105.Amano, M., Fukata, Y., and Kaibuchi, K. 2000. Regulation and functions of Rho-associated kinase. Exp. Cell Res. 261:44-51.

106.Sin, W.-C., Chen, X.-Q., Leung, T., and Lim, L. 1998. RhoA-Binding Kinase alpha Translocation Is Facilitated by the Collapse of the Vimentin Intermediate Filament Network. Mol. Cell. Biol. 18:6325-6339.

107. Sahlgren, C.M., et al. 2003. Cdk5 regulates the organization of Nestin and its association with p35. Mol. Cell. Biol. 23:5090-5106.

108.Sahlgren, C.M., et al. 2006. A nestin scaffold links $\mathrm{Cdk} 5 / \mathrm{p} 35$ signaling to oxidant-induced cell death. EMBO J. 25:4808-4819.

109.Bieberich, E., MacKinnon, S., Silva, J., Noggle, S. and Condie, B.G. 2003. Regulation of cell death in mitotic neural progenitor cells by asymmetric distribution of prostate apoptosis response 4 (PAR-4) and simultaneous elevation of endogenous ceramide. J. Cell Biol. 162:469-479.

110.He, T., Stepulak, A., Holmstrom, T.H., Omary, M.B., and Eriksson, J.E. 2002. The intermediate filament protein keratin 8 is a novel cytoplasmic substrate for c-Jun $\mathrm{N}$-terminal kinase. J. Biol. Chem. 277:10767-10774.

111.Helfand, B.T., Chou, Y.H., Shumaker, D.K., and Goldman, R.D. 2005. Intermediate filament proteins participate in signal transduction. Trends Cell Biol. 15:568-570.

112.Perlson, E., et al. 2005. Vimentin-dependent spatial translocation of an activated MAP kinase in injured nerve. Neuron. 45:715-726.

113.Caulin, C., Ware, C.F., Magin, T.M., and Oshima, R.G. 2000. Keratin-dependent, epithelial resistance to tumor necrosis factor-induced apoptosis. J. Cell Biol. 149:17-22.

114.Gilbert, S., Loranger, A., Daigle, N., and Marceau, N. 2001. Simple epithelium keratins 8 and 18 pro- 
vide resistance to Fas-mediated apoptosis. The protection occurs through a receptor-targeting modulation. J. Cell Biol. 154:763-773.

115.Inada, H., et al. 2001. Keratin attenuates tumor necrosis factor-induced cytotoxicity through association with TRADD. J. Cell Biol. 155:415-426.

116.Tong, X., and Coulombe, P.A. 2006. Keratin 17 modulates hair follicle cycling in a TNFalphadependent fashion. Genes Dev. 20:1353-1364.

117. Dinsdale, D., Lee, J.C., Dewson, G., Cohen, G.M., and Peter, M.E. 2004. Intermediate filaments control the intracellular distribution of caspases during apoptosis. Am. J. Pathol. 164:395-407.

118.Gilbert, S., Loranger, A., and Marceau, N. 2004. Keratins modulate c-Flip/extracellular signal-regulated kinase 1 and 2 antiapoptotic signaling in simple epithelial cells. Mol. Cell. Biol. 24:7072-7081.

119. Ku, N.O., Liao, J., and Omary, M.B. 1998. Phosphorylation of human keratin 18 serine 33 regulates binding to 14-3-3 proteins. EMBOJ. 17:1892-1906. 120.Liao, J., and Omary, M.B. 1996. 14-3-3 proteins associate with phosphorylated simple epithelial keratins during cell cycle progression and act as a solubility cofactor. J. Cell Biol. 133:345-357.

121. Toivola, D.M., et al. 2001. Disturbances in hepatic cell-cycle regulation in mice with assembly-deficient keratins 8/18. Hepatology. 34:1174-1183.

122. Kim, S., Wong, P., and Coulombe, P.A. 2006. A keratin cytoskeletal protein regulates protein synthesis and epithelial cell growth. Nature. 441:362-365.

123. Ku, N.O., et al. 1998. Mutation of a major keratin phosphorylation site predisposes to hepatotoxic injury in transgenic mice. J. Cell Biol. 143:2023-2032.

124.Ku, N.O., and Omary, M.B. 2006. A disease- and phosphorylation-related nonmechanical function for keratin 8. J. Cell Biol. 174:115-125.

125.Toivola, D.M., Zhou, Q., English, L.S., and Omary, M.B. 2002. Type II keratins are phosphorylated on a unique motif during stress and mitosis in tissues and cultured cells. Mol. Biol. Cell. 13:1857-1870.

126.Gonzales, M., et al. 2001. Structure and function of a vimentin-associated matrix adhesion in endothelial cells. Mol. Biol. Cell. 12:85-100.

127. Tsuruta, D., and Jones, J.C. 2003. The vimentin cytoskeleton regulates focal contact size and adhesion of endothelial cells subjected to shear stress. J. Cell Sci. 116:4977-4984.

128.Ivaska, J., et al. 2005. PKCepsilon-mediated phosphorylation of vimentin controls integrin recycling and motility. EMBO J. 24:3834-3845.

129.Szeverenyi, I., et al. 2008. The Human Intermediate Filament Database: comprehensive information on a gene family involved in many human diseases. Hum. Mutat. 29:351-360.

130. Müller, M., et al. 2009. Dominant cataract formation in association with a vimentin assembly-disrupting mutation. Hum. Mol. Genet. 18:1052-1057. 